\title{
Aprendizaje de Vectores Euclidianos utilizando un Sistema de Realidad Aumentada
}

\author{
Angel Chi-Poot, Anabel Martin-Gonzalez, Victor Menendez-Dominguez y Arturo \\ Espinosa-Romero \\ Facultad de Matemáticas, Universidad Autónoma de Yucatán, Periférico Norte, \\ 97117 Mérida, México \\ angel.linux91@gmail.com, \{amarting, mdoming, eromero\}@uady.mx
}

\begin{abstract}
Resumen. La realidad aumentada es una tecnología que puede ser implementada como una herramienta práctica y eficiente para mejorar las técnicas de aprendizaje en estudiantes de bachillerato. En este artículo, presentamos resultados recientes de investigación en desarrollo sobre un sistema de realidad aumentada para asistir el aprendizaje de vectores euclidianos en física para estudiantes. Para comprender tales conceptos físicos, el sistema proyecta componentes virtuales en el mundo real, y se controla a través de una interfaz interactiva con el cuerpo del usuario. En este trabajo, se describe el prototipo, su interfaz de usuario y su evaluación preliminar.
\end{abstract}

Palabras clave: Augmented reality, physics education, educational technology.

\section{Introducción}

Los avances constantes en tecnologías de la información y comunicación (TICs) han tenido un impacto positivo en la educación, motivando la implementación de nuevas herramientas, aplicaciones, medios y ambientes educativos para promover el aprendizaje.

Las innovaciones tecnológicas tales como escenarios virtuales, dispositivos móviles inalámbricos, plataformas digitales de enseñanza, realidad virtual y aumentada, incrementan el interés y motivación de los estudiantes, así como su experiencia de aprendizaje [1], [2], [3].

La realidad aumentada (RA) es una tecnología que mejora la percepción visual del usuario a través de la superimposición de objetos virtuales, generados por computadora, al mundo real [4], [5]. Opuesto a la realidad virtual (RV), donde el usuario se encuentra completamente inmerso en un ambiente virtual generado por computadora, la realidad aumentada permite al usuario observar directamente el mundo real pero agregando objetos virtuales. De esta manera, la RA complementa la realidad en lugar de remplazarla totalmente.

Según Cai et al., un ambiente educativo basado en la RA va de acuerdo con varias teorías del aprendizaje. Una de ellas, por ejemplo, se centra en que el aprendizaje es el resultado de asociaciones formadas entre estímulos y respuestas. Por el lado del constructivismo, una plataforma de enseñanza basada en RA provee a los estudiantes 
con herramientas y escenarios de construcción de modelos, diseñados para ser fácilmente utilizados por ellos [6].

Existen varias aplicaciones de realidad aumentada propuestas para asistir diferentes áreas académicas. En astronomía, por ejemplo, Shelton y Hedley, desarrollaron un sistema de RA para enseñar la relación entre el Sol y la Tierra a través del uso de formas tridimensionales (3D) virtuales del astro y del planeta [7]. En química, Fjeld et al., implementaron una interfaz de usuario tangible, denominada 'Augmented Chemistry (AC)', para mostrar a los estudiantes modelos moleculares tridimensionales a través de RA [8], [9]. Por otro lado, Maier et al., evaluaron una interfaz de usuario 3D de realidad aumentada para mejorar el entendimiento de la química molecular [10]. En biología, Blum et al., desarrollaron el 'Mirracle', un sistema de realidad aumentada que funciona como un espejo virtual que permite entender la estructura y funcionalidad de la anatomía y órganos internos del cuerpo humano de manera intuitiva [11]. En el área de matemáticas y geometría, Kaufmann y Schmalstieg, diseñaron el sistema multiusuario Construct3D para construir formas geométricas virtuales [12]. En física, Duarte et al., utilizaron la RA para mejorar el aprendizaje de propiedades cinemáticas [13].

La enseñanza de las ciencias básicas ha sido siempre una problemática presente en la educación media superior, especialmente la de la física, principalmente por la necesidad de que el estudiante adquiera un nivel cognitivo tal que le permita inferir los aspectos abstractos que están detrás de situaciones que se presentan en la vida real. Esto se hace más patente en la física de vectores, que tiene una importante relación con la dinámica, la mecánica de fluidos, la fuerza, entre otros. En México, el aprendizaje de vectores euclidianos y, en particular, sus operaciones aritméticas son vistos de manera implícita (graficando líneas en un plano cartesiano bidimensional) y en una medida gradual, acorde a las capacidades intelectuales de los estudiantes. Sin embargo, el uso de nuevas tecnologías, como la realidad aumentada, podría enriquecer las limitantes de los actuales recursos educativos para facilitar el aprendizaje de dichas temáticas.

En matemáticas, física e ingeniería, los vectores euclidianos son objetos geométricos que caracterizan cantidades físicas que poseen magnitud y dirección (ej. fuerza, velocidad, aceleración), contrarias a cantidades escalares que no poseen dirección (ej. tiempo, temperatura, distancia). A dichos vectores se les puede aplicar una diversidad de operaciones matemáticas. Una de ellas es la adición, que es la suma de vectores, y puede representar, por ejemplo, la red de fuerzas que experimenta un objeto, es decir, el vector suma de todas las fuerzas individuales interactuando sobre ese objeto. La sustracción es otra operación, la cual puede ser vista como una adición con un vector negativo (operación opuesta a la adición). El producto cruz (también llamado vector producto), $\mathbf{a} \times \mathbf{b}$, es un vector perpendicular a ambos vectores $\mathbf{a} \mathbf{y} \mathbf{b}, \mathbf{y}$ se define como:

$$
\mathbf{a} \times \mathbf{b}=\|\mathbf{a}\|\|\mathbf{b}\| \sin (\theta) \mathbf{n},
$$

donde $\theta$ es la medida del ángulo entre $\mathbf{a}$ y $\mathbf{b}$, y $\mathbf{n}$ es un vector unitario perpendicular a ambos vectores a y b. La magnitud del producto cruz es el área del paralelogramo de lados a y b. La dirección del producto cruz es ortogonal al plano que contiene dicho paralelogramo. 
El objetivo de este trabajo es presentar el desarrollo de una herramienta de realidad aumentada diseñada para permitir al profesor el uso de técnicas modernas para la enseñanza de conceptos de la física (ej. propiedades y operaciones vectoriales), ayudando, de esta manera, a los estudiantes a tener un aprendizaje más completo a través de ambientes de realidad aumentada.

\section{Métodos}

\subsection{Configuración del Sistema}

El sistema de realidad aumentada presentado en este trabajo ha sido desarrollado para apoyar el aprendizaje de conceptos de física en el aula de clases. En particular, se enfoca en el entendimiento de las propiedades relacionadas a los vectores euclidianos (magnitud y dirección), y algunas operaciones de vectores (suma, resta y producto cruz).

Nuestro sistema consiste de un monitor externo, una cámara de color y una de profundidad (ver Fig. 1). El monitor permite la visualización del mundo real y los objetos virtualmente aumentados. Las cámaras de color y profundidad forman parte del sensor Kinect de Microsoft ${ }^{\mathrm{TM}}$, desarrollado para el juego de consola Xbox 360. Dicho sensor habilita el uso de señas y movimientos del cuerpo como interfaz de control del sistema. La estación central para el cómputo en general consiste en un procesador Intel ${ }^{\mathrm{TM}}$ Core $\mathrm{i7}-3630 \mathrm{QM}$ con $8 \mathrm{~GB}$ de RAM y una tarjeta de gráficos dedicada Nvidia ${ }^{\mathrm{TM}}$ GeForce GT 640MB.
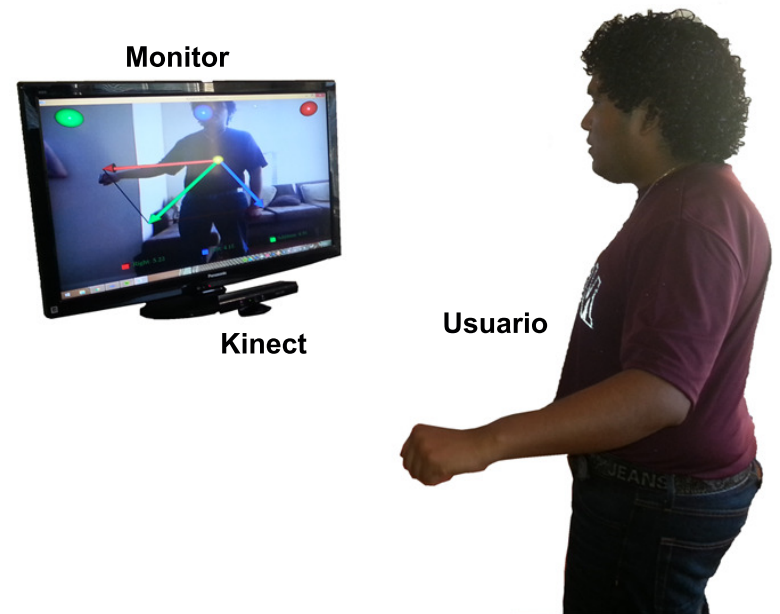

Fig. 1. Configuración del sistema de realidad aumentada. El usuario puede visualizar los vectores virtuales en la pantalla mientras mueve las manos para cambiar magnitudes y direcciones. 


\subsection{Interfaz del Usuario}

La posición del usuario es rastreada, constantemente, con la cámara de profundidad utilizando las librerías NITE de rastreo del esqueleto (www.openni.org). Las coordenadas 3D del mundo real obtenidas por el Kinect son transformadas al sistema de coordenadas 2D del monitor a través de las librerías de OpenNI. Finalmente, los gráficos generados son utilizando las librerías de OpenGL (www.opengl.org). Ver Fig. 2.

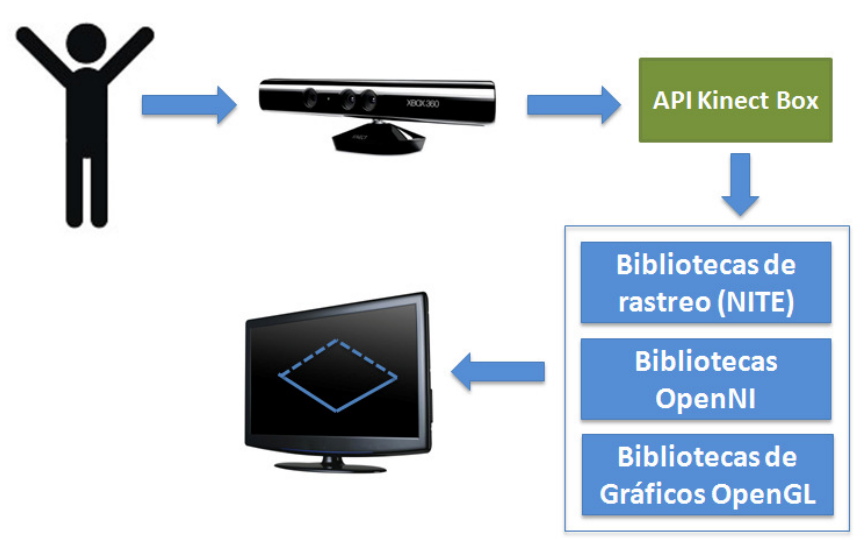

Fig. 2. Arquitectura funcional del sistema de RA.

El sistema de RA permite al usuario seleccionar una de tres operaciones de vectores diferentes: adición, sustracción o producto cruz. Cada modalidad puede ser elegida tocando, durante tres segundos, botones virtuales desplegados en una ubicación 3D fija visualizados en el monitor.

Las coordenadas 3D del torso del individuo, obtenidas con el Kinect, son usadas para dar lugar al origen común para formar dos vectores concurrentes i y d. La posición 3D de la mano izquierda y de la mano derecha son continuamente rastreadas para obtener los puntos finales de los vectores i y d correspondientes. El vector resultante de cada operación es generado virtualmente y visualizado en el ambiente de RA. Asimismo, al cambiar de posición las manos, el sistema le permite observar, dinámicamente, diferentes magnitudes y direcciones de los vectores. La información de las magnitudes correspondientes a cada uno de los vectores visualizados se despliega como texto en la parte inferior de la pantalla.

Inicialmente, el sensor Kinect requiere ser calibrado para el usuario con una postura específica del cuerpo con el fin de que el sensor rastree correctamente el esqueleto del individuo (pose de letra griega 'Psi', $\Psi$ ). Para ello, la persona tiene que pararse derecho frente a las cámaras del Kinect con las manos por encima de la cabeza (en escuadra). Una vez que el sensor ha identificado al usuario, el sistema de RA inicia el rastreo de los puntos de interés (torso y manos). 


\section{Resultados}

La visualización final de la adición de vectores se puede observar en la Fig. 3. Las posiciones de las manos izquierda (azul) y derecha (rojo) del usuario definen los puntos terminales de los dos vectores i y d, correspondientes, con los cuales la suma será calculada. El vector resultante de la suma se puede visualizar en el vector virtual color verde. La posición del torso del usuario se identifica con una esfera amarilla, indicando el origen común de los vectores. Para esta operación vectorial, el usuario puede mover las manos en diferentes posiciones para cambiar de magnitud y dirección los vectores, observando, de esta manera, diferentes resultados de adición.

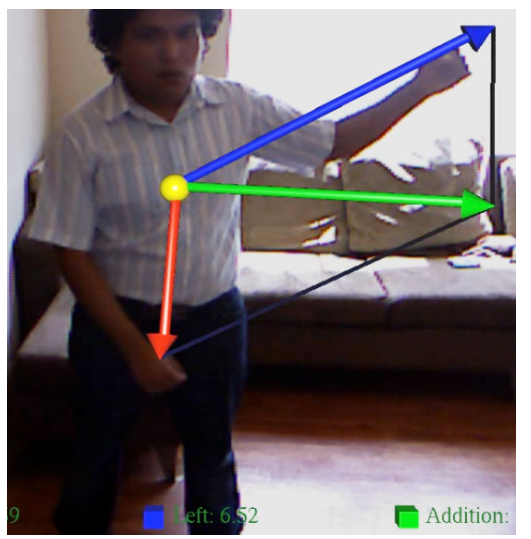

Fig. 3. Vista aumentada del sistema de RA para la operación de adición de vectores.

La visualización de la sustracción de vectores se presenta en la Fig. 4. El sistema resta el vector $\mathbf{i}$ en color azul del vector $\mathbf{d}$ en color rojo. El vector resultado de esta operación se visualiza en color cian.

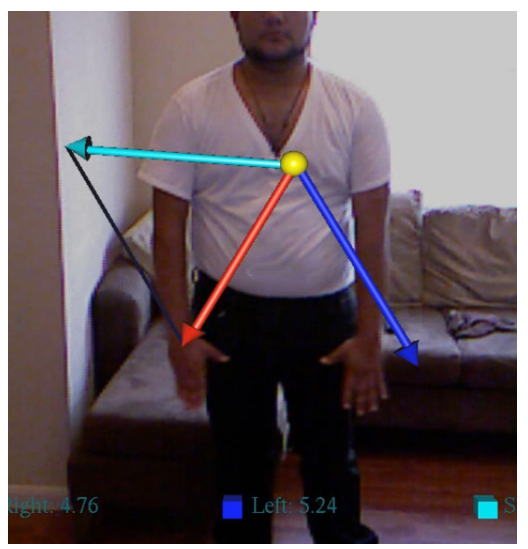

Fig. 4. Vista aumentada del sistema de RA para la operación de resta de vectores. 
La visualización del producto cruz de vectores se muestra en la Fig. 5. El vector producto-cruz (en color naranja) es el resultado de aplicar la ecuación 1 a los vectores i (azul) y d (rojo). En la visualización aumentada se puede observar la propiedad de ortogonalidad del producto cruz.

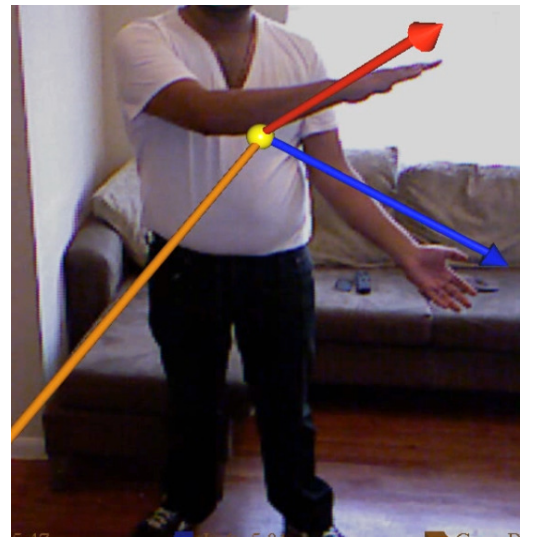

Fig. 5. Vista aumentada del sistema de RA para la operación producto cruz de vectores.

Evaluamos el sistema de realidad aumentada para enseñar operaciones vectoriales con 12 estudiantes de enseñanza media-superior y un profesor mediante una encuesta de opinión abierta. La retroalimentación de los sujetos fue muy positiva y motivadora, haciendo notar desventajas menores del sistema, como por ejemplo, la pérdida de rastreo de la posición de las manos cuando éstas estaban fuera del rango del sensor. Durante los experimentos, los usuarios no requirieron de una explicación exhaustiva de cómo utilizar el sistema.

\section{Discusión}

Como podemos observar en las Fig. 3, 4 y 5, el usuario puede crear vectores de varias magnitudes y direcciones utilizando únicamente las manos de una manera dinámica. El usuario puede trasladar las manos a diferentes posiciones tridimensionales dentro del área de rastreo del sensor Kinect para generar los vectores virtuales con varias magnitudes y direcciones.

La interacción observable de los sujetos con el sistema de RA fue muy interesante. Los usuarios analizaban los vectores virtuales generados que ellos mismos controlaban con las manos. Intentaron varias posturas de cuerpo y manos para ver y entender los efectos de la salida de cada operación. Al cruzar las manos, observaron como el vector producto-cruz cambió su dirección $180^{\circ}$. Al cambiar las magnitudes y/o direcciones de los vectores, los estudiantes observaron las diferencias en el vector resultante de la adición y sustracción de vectores. La mayoría de los estudiantes expresaron que el sistema es una buena herramienta para aprender conceptos de física 
como son los vectores y sus respectivas operaciones. Asimismo, mencionaron que la interfaz del sistema es bastante fácil de utilizar, entender y aprender interactuando.

En los métodos tradicionales de enseñanza, los materiales didácticos estáticos (ej. pizarrón, proyección, láminas) están restringidos para presentar información dinámica, como, por ejemplo, el movimiento continuo [14], [15], a diferencia con nuestro sistema de RA que permite al usuario, inclusive, interactuar con los elementos de estudio. Asimismo, nuestro sistema propuesto puede ser eficiente para la fácil comprensión de conceptos y propiedades existentes en un espacio tridimensional, como es el caso del producto cruz.

Como trabajo a futuro, un sistema de RA para la enseñanza de vectores más completo, necesitaría considerar más propiedades y operaciones de vectores (ej. vector de proyección, producto punto); asimismo, analizar las posibles mejoras a la interfaz de usuario para hacer más sencillo aún su uso.

Aún cuando los métodos actuales de aprendizaje son frecuentemente eficaces, existe un constante interés por desarrollar métodos pedagógicos más útiles y prácticos para mejorar las experiencias de enseñanza.

\section{Conclusión}

Mientras las tecnologías continúen su progreso, los educadores se encuentran en constante búsqueda de herramientas pedagógicas más eficientes para mejorar el proceso de aprendizaje de los estudiantes.

Este trabajo presenta el desarrollo de un sistema de realidad aumentada basado en una interfaz de interacción con el cuerpo para asistir el entendimiento y aprendizaje de propiedades y operaciones de vectores en física. Los usuarios fueron capaces de crear vectores virtualmente con diferentes magnitudes y direcciones, así como visualizar sus propiedades y operaciones. Asimismo, todos tuvieron una reacción positiva hacia el uso de un sistema de RA para el aprendizaje de vectores euclidianos.

Agradecimientos. Este proyecto fue financiado por el Programa de Mejoramiento del Profesorado (PROMEP) 2013 - 103.5/13/6979.

\section{Referencias}

1. Lee, K.: Augmented reality in education and training. TechTrends 56(2), 13--21(2012)

2. Roussos, M., Johnson, A., Moher, T., Leigh, J., Vasilakis, C., Barnes, C.: Learning and building together in an immersive virtual world. Presence 8(3), 247--263 (1999)

3. Nincarean, D., Alia, M.B., Halim, N.D.A., Rahman, M.H.A.: Mobile augmented reality: The potential for education. Procedia Soc. Behav. Sci. 103, 657--664 (2013)

4. Azuma, R., Baillot, Y., Behringer, R., Feiner, S., Julier, S., MacIntyre, B.: Recent advances in augmented reality. IEEE Comput. Graph. Appl. 21(6), 34--47 (2001)

5. Azuma, R.: A survey of augmented reality. Presence: Teleoperators and Virtual Environments 6(4), 355--385 (1997) 
6. Cai, S., Chiang, F.K., Wang, X.: Using the augmented reality 3D technique for a convex imaging experiment in a physics course. Int. J. Eng. Educ. 29(4), 856--865 (2013)

7. Shelton, B.E., Hedley, N.R.: Using augmented reality for teaching Earth-Sun relationships to undergraduate geography students. In: The First IEEE International Augmented Reality Toolkit Workshop (2002)

8. Fjeld, M., Voegtli, B.M.: Augmented chemistry: An interactive educational workbench. In: ISMAR. pp. 259--260 (2002)

9. Fjeld, M., Fredriksson, J., Ejdestig, M., Duca, F., Býtschi, K., Voegtli, B.M., Juchli, P.: Tangible user interface for chemistry education: comparative evaluation and redesign. In: Rosson, M.B., Gilmore, D.J. (eds.) CHI. pp. 805--808. ACM (2007)

10. Maier, P., Klinker, G.: Augmented chemical reactions: 3D interaction methods for chemistry. International Journal of Online Engineering 9, 80--82 (2013)

11. Blum, T., Kleeberger, V., Bichlmeier, C., Navab, N.: mirracle: An augmented reality magic mirror system for anatomy education. In: IEEE Virtual Reality Short Papers and Posters. pp. 115--116 (2012)

12. Kaufmann, H., Schmalstieg, D.: Mathematics and geometry education with collaborative augmented reality. In: ACM SIGGRAPH 2002 Conference Abstracts and Applications. pp. 37--41. ACM (2002)

13. Duarte, M., Cardoso, A., Lamounier Jr., E.: Using augmented reality for teaching physics. In: WRA 2005 - II Workshop on Augmented Reality. pp. 1--4 (2005)

14. Craig, A., McGrath, R.: Augmenting Science Texts with Inexpensive Interactive 3D Illustrations (2007)

15. Kühl, T., Scheiter, K., Gerjets, P., Gemballa, S.: Can differences in learning strategies explain the benefits of learning from static and dynamic visualizations? Computers \& Education 56(1), 176--187 (2011) 